\title{
Rotational Diffusion of Mitochondrial ADP/ATP Carrier Studied by Saturation-Transfer Electron Spin Resonance
}

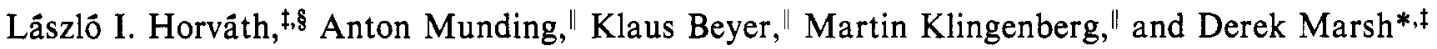 \\ Abteilung Spektroskopie, Max-Planck-Institut für Biophysikalische Chemie, D-3400 Göttingen, Federal Republic of Germany, \\ and Institut für Physiologische Chemie, Physikalische Biochemie und Zellbiologie, Universität München, D-8000 München 2 , \\ Federal Republic of Germany \\ Received March 25, 1988; Revised Manuscript Received July 19, 1988
}

\begin{abstract}
The rotational mobility of the mitochondrial ADP/ATP carrier has been studied solubilized in Triton micelles, reincorporated in phospholipid liposomes, and in mitochondria. Spin-labeled analogues of the noncovalent inhibitors carboxyatractyloside and atractyloside were found to be strongly immobilized when bound to the carrier [Munding, A., Beyer, K., \& Klingenberg, M. (1983) Biochemistry 22, 1941-1947], such that saturation-transfer electron spin resonance spectroscopy could be used to study the rotational motion of the protein. Spin-labeled maleimide covalently bound to the carrier was found to have independent segmental motion and hence to be unsuitable for studies of protein rotation. The ESR spectra of the carboxyatractyloside and atractyloside spin labels were found to contain a second component from label in the lipid or detergent, necessitating the use of the saturation-transfer ESR integral method [Horväth, L. I., \& Marsh, D. (1983) J. Magn. Reson. 54, 363-373] to analyze the rotational motion of the label component bound to the protein. Effective rotational correlation times obtained from integration of the high-field region of the spectrum were lower than those obtained from the total spectral integral, indicating strongly anisotropic rotational diffusion of the carrier in the vesicular and membrane systems, with the spin-label $z$ axis oriented preferentially perpendicular to the rotation axis. Using the results of spectral line-shape simulations for highly anisotropic rotation [Robinson, B. H., \& Dalton, L. R. (1980) J. Chem. Phys. 72, 1312-1324], it was estimated that the correlation times for the axial rotational diffusion of the carrier were $\tau_{\mathrm{R} \mid}=1-5 \mu \mathrm{s}$ in phospholipid liposomes and 2-20 $\mu \mathrm{s}$ in mitochondria, at temperatures of 30 and $0{ }^{\circ} \mathrm{C}$, respectively. These values are upper estimates. Comparison with theoretical estimates of the rotational correlation time based on hydrodynamic models suggests that the carrier protein is a dimer when reincorporated in phospholipid liposomes and in detergent and that it undergoes slower rotational diffusion due to interaction with other proteins in the mitochondrial membrane.
\end{abstract}

$\mathbf{T}_{\text {h }}$ 5 -triphosphate across the inner mitochondrial membrane is a key transport process which is effected by a membrane-bound protein, the ADP/ATP carrier (AAC) ${ }^{1}$ [for a review, see Klingenberg (1985)]. The carrier is selectively inhibited by a plant glycoside, carboxyatractylate, which binds with high affinity to the AAC. Abundant evidence indicates that carboxyatractylate binds to the same site as ADP and ATP. The spin-labeled derivative CATSL (I) also binds with high affinity

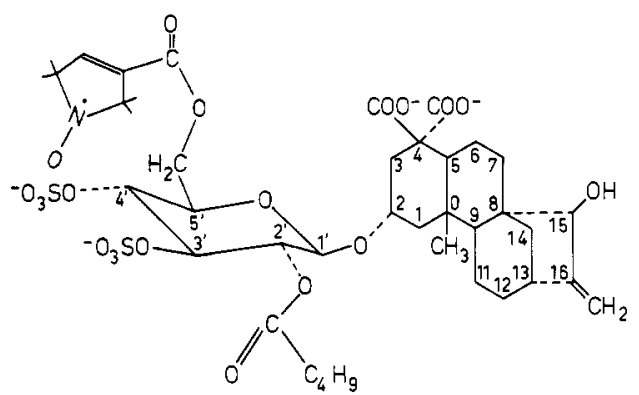

I

to the AAC. Detailed ESR measurements showed that CATSL, when bound to the carrier either in the mitochondrial membrane or in the isolated solubilized state or when incor-

\footnotetext{
Max-Planck-Institut für Biophysikalische Chemie, Göttingen.

Permanent address: Institute of Biophysics, Biological Research Centre, Szeged, Hungary.

"Universität München.
}

porated into liposomes, exhibits an unusually large hyperfine splitting and narrow line widths, indicating strong immobilization of the spin label (Munding et al., 1983). These results do not exclude a slow overall rotational motion of the protein, since such low mobilities are beyond the limits of sensitivity of conventional ESR spectroscopy (typically $10^{-7} \mathrm{~s}$; Marsh, 1985). With a different approach, Lauquin et al. (1977) have used spin-labeled atractyloside with the nitroxide group attached to a covalently linked acyl chain to probe lipid-protein interactions and the form of the intramembranous surface of the carrier.

In the present study, the rotational diffusion of the AAC was investigated by saturation-transfer ESR (STESR) spectroscopy, which extends the limits of the sensitivity range of the spin-label ESR method from rotational correlation times of $10^{-7} \mathrm{~s}$ down to $10^{-3} \mathrm{~s}$ (Thomas et al., 1976). It is desirable to have more detailed information about the protein mobility to determine the freedom of motion and molecular interactions in the native mitochondrial membrane (Müller et al., 1982).

\footnotetext{
${ }^{1}$ Abbreviations: ATR, atractylate; CAT, carboxyatractylate ATRSL, ATR spin labeled with 2,2,5,5-tetramethyl-1-oxy-3-pyrroline3-carboxylic acid; CATSL, CAT spin labeled with 2,2,5,5-tetramethyl1-oxy-3-pyrroline-3-carboxylic acid; 5-MSL, 3-maleimido-2,2,5,5-tetramethylpyrrolidinyl-1-oxy; AAC, ADP/ATP carrier; ESR, electron spin resonance; STESR, saturation-transfer ESR; $v_{1}$, first harmonic ESR absorption signal detected in-phase with respect to the field modulation; $v_{2}{ }^{\prime}$, second harmonic ESR absorption signal detected $90^{\circ}$ out-of-phase with respect to the field modulation; PC, egg phosphatidylcholine; PE, egg phosphatidylethanolamine; $\mathrm{CL}$, bovine heart cardiolipin.
} 
For example, the question as to whether the AAC is associated with other proteins, such as the ATP synthetase with which it is functionally linked, can be addressed by this investigation. Additionally, mobility data may be used further to interpret and to substantiate previously reported evidence on the shape and on the oligomeric state of the AAC (Hakenberg \& Klingenberg, 1980).

The application of STESR is, however, not straightforward in this system because multicomponent spectra are observed with the CATSL (Munding et al., 1983) which cannot be analyzed in terms of the usual STESR diagnostic line-height ratios. A way to overcome this problem is the use of the so-called STESR integral method which was shown successfully to discriminate multicomponent spectra of very different correlation times (Evans, 1981; Horvăth \& Marsh, 1983). Here we present STESR integral data for the rotational mobility of the mitochondrial ADP/ATP carrier in its Triton-solubilized, liposome-reincorporated, and native form. The results are used to provide information about the aggregation state of the protein in detergent and in lipid liposomes of various compositions, as well as about interactions with the other proteins of the inner mitochondrial membrane to which the carrier is functionally linked. The difference in measured rotational mobility with CATSL and with a covalently bound maleimide spin label is also discussed.

\section{Materials and Methods}

Spin-labeled carboxyatractylate (structure I) and atractylate were prepared as described in Munding et al. (1983). Spinlabeled maleimide (5-MSL) was obtained from Syva, Palo Alto, CA. Phospholipids were prepared from egg yolk by standard procedures (Wells \& Hanahan, 1969). Beef heart cardiolipin was obtained from Sigma, St. Louis, MO.

Beef heart mitochondria were isolated according to Blair (1967) and stored at $-176^{\circ} \mathrm{C}$. Spin labeling of the mitochondria by CATSL was performed as described previously (Munding et al., 1983). The ATR-carrier complex was isolated in Triton X-100 from ATR-loaded mitochondria (Aquila \& Klingenberg, 1982). ATR was exchanged for CATSL and the CATSL-protein complex was reincorporated into phospholipid liposomes according to a one-step procedure as detailed previously (Beyer \& Klingenberg, 1983; Munding et al., 1983). In a typical experiment about $3.5 \mathrm{mg}$ of the CATSL-labeled protein in $0.5 \mathrm{~mL}$ of $10 \% \mathrm{w} / \mathrm{v}$ Triton X-100 was added to $30 \mathrm{mg}$ of phospholipid at $4{ }^{\circ} \mathrm{C}$. Excess Triton was removed by addition of $3.8 \mathrm{~g}$ of wet Amberlite XAD-2 beads. The phospholipid-protein liposomes formed were centrifuged at $136000 \mathrm{~g}$ for $1 \mathrm{~h}$.

Spin labeling of the ADP/ATP carrier with 5-MSL maleimide spin label was performed as described by Munding et al. (1987).

ESR spectra were recorded on a Varian E-12 Century Line spectrometer interfaced to a PDP 11/10 laboratory computer. Aliquots of the membrane suspensions in argon-saturated buffer were filled into $1-\mathrm{mm}$ diameter glass capillaries flushed with argon and centrifuged at full speed on a bench centrifuge for $10 \mathrm{~min}$. Excess supernatant was removed to obtain pellets of 5-mm length under argon. This recommended sample configuration was used in all STESR experiments (Fajer \& Marsh, 1982; Hemminga et al., 1984). Sample capillaries were centered in a standard 4-mm quartz tube containing light silicone oil for thermal stability; temperature was regulated with a pure nitrogen gas flow system. Conventional, in-phase, absorption spectra ( $v_{1}$ display) were recorded at a modulation frequency of $100 \mathrm{kHz}$ and modulation amplitude of $1.6 \mathrm{G} \mathrm{p}-\mathrm{p}$, at the same microwave power as used for the STESR ex-

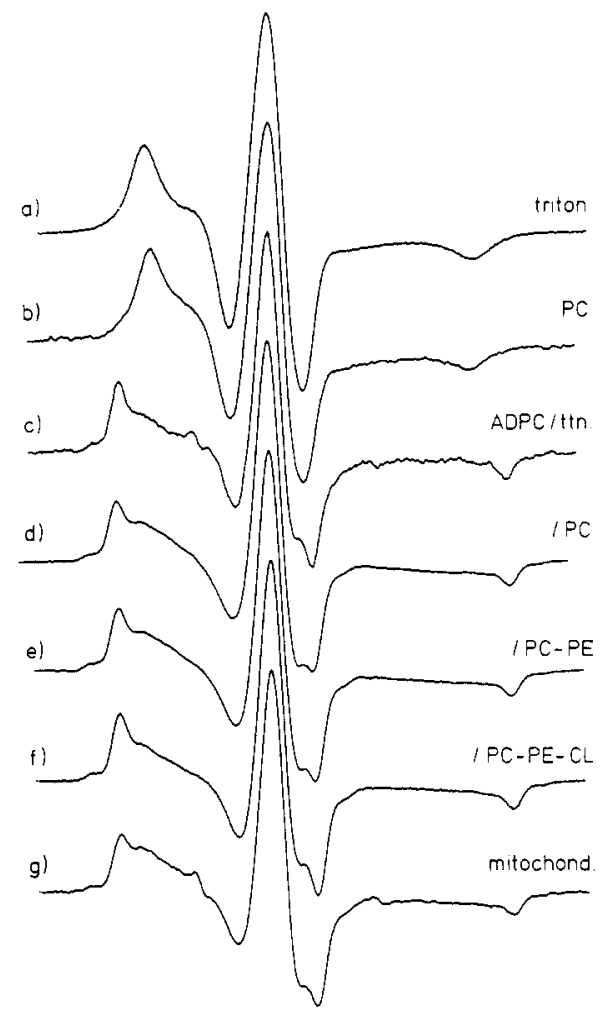

FIGURE 1: Conventional, in-phase ESR spectra ( $v_{1}$ display) of spinlabeled carboxyatractylate (CATSL) in various environments at 0 ${ }^{\circ} \mathrm{C}$ : (a) in Triton micelles (35\% Triton X-100 in water); (b) in egg phosphatidylcholine liposomes; (c) bound to Triton-solubilized ADP/ATP carrier; (d-f) bound to reincorporated ADP/ATP carrier in (d) phosphatidylcholine, (e) phosphatidylcholine-phosphatidylethanolamine $(6: 4 \mathrm{w} / \mathrm{w})$, and (f) phosphatidylcholine-phosphatidylethanolamine-cardiolipin (5.5:3.5:1.0 w/w) liposomes; $(\mathrm{g})$ bound to native mitochondrial ADP/ATP carrier. Total scan range $=100 \mathrm{G}$.

periments. STESR spectra were recorded in the second harmonic, $90^{\circ}$ out-of-phase, absorption mode ( $v_{2}{ }^{\prime}$ display) at a modulation frequency of $50 \mathrm{kHz}$ and modulation amplitude of $5 \mathrm{G} \mathrm{p}-\mathrm{p}$. All STESR measurements were performed at a microwave power which gave an average $H_{1}$ field over the sample of $0.25 \mathrm{G}$, according to the recommended protocol (Fajer \& Marsh, 1982; Hemminga et al., 1984). Details of the calibration of our spectrometer, along with line-height and STESR integral calibrations, are described elsewhere (Horväth $\&$ Marsh, 1983, 1988). Spectral subtractions of the conventional, in-phase spectra were performed with an interactive graphic display terminal using software written by Dr. W. Möller of this Institute.

\section{RESULTS}

Conventional, in-phase ESR spectra of the CATSL label bound to Triton-solubilized, liposome-reincorporated, and native mitochondrial ADP/ATP carrier are shown in Figure 1 (spectra $\mathrm{c}-\mathrm{g}$ ). For comparison, the spectra of CATSL in Triton X-100 micelles and in egg phosphatidylcholine liposomes are given in spectra a and b of Figure 1, respectively. The liposome-bound CATSL spectrum was obtained by quenching the overlapping absorption of freely tumbling CATSL in water by the addition of $200 \mathrm{mM} \mathrm{NiCl}$. The spectra were recorded at $0^{\circ} \mathrm{C}$. All spectra from the carrier-containing samples are complex, showing at least two spectral components; occasionally, a trace of freely tumbling CATSL was also observed (e.g., spectra $\mathrm{c}$ and g). One component has an unusually large maximum hyperfine splitting $\left(2 A_{\max } \sim 70 \mathrm{G}\right)$ and has been assigned to CATSL molecules selectively bound to the inhibitor binding site, as demonstrated 


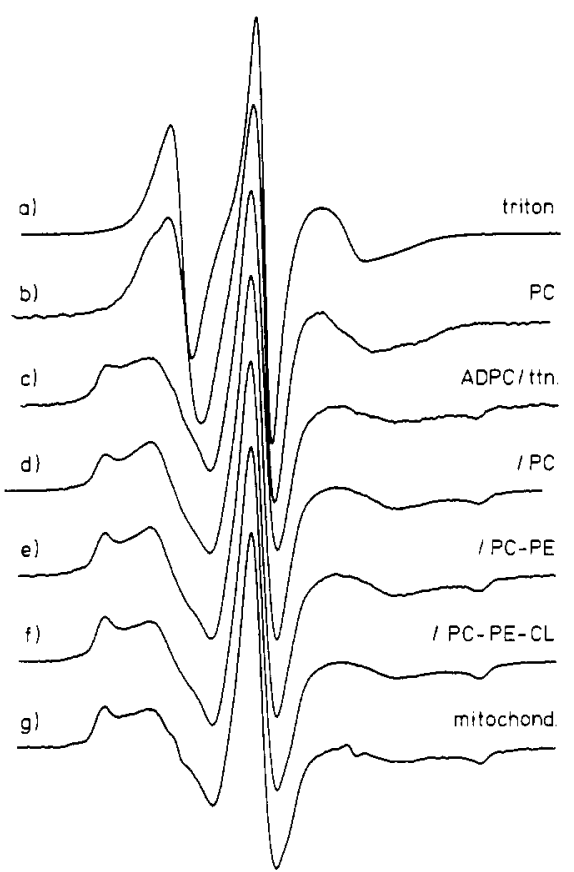

FIGURE 2: Conventional, in-phase ESR spectra ( $v_{1}$ display) of CATSL in various environments at $30^{\circ} \mathrm{C}$. $(\mathrm{a}-\mathrm{g})$ as defined in Figure 1 . Total scan width $=100 \mathrm{G}$.

by competition experiments with unlabeled CAT (Munding et al., 1983). The second component has a smaller maximum splitting and closely resembles the micellar- or liposome-bound CATSL. In the spectrum of the Triton-solubilized protein (Figure 1c) a component matching the Triton-alone spectrum (Figure 1a) is clearly seen overlapping the sharp outer peaks from the immobilized component. In the spectra of the membrane-bound carrier (Figure 1d-g), the second component has a yet smaller splitting than the spectrum from egg phosphatidylcholine alone (Figure 1b), indicating a higher degree of lipid mobility in the lipid-protein systems. A dramatic influence of increasing temperature on the line narrowing of the mobile component was previously reported, whereas until $30^{\circ} \mathrm{C}$ the hyperfine splitting of the immobilized component remained unchanged. Only above $30^{\circ} \mathrm{C}$ did the hyperfine splitting of this component decrease, reflecting mobility within the limits of conventional ESR (Munding et al., 1983). Thus the spectra of the more mobile component are strongly temperature dependent, in contrast to the immobilized component which lies at the limits of motional sensitivity of conventional ESR spectroscopy. This is seen from the spectra recorded at $30^{\circ} \mathrm{C}$ given in Figure 2. The two spectral components are distinguished even more clearly at this temperature: the more mobile component is now extensively motionally narrowed, whereas the immobilized component retains close to its maximum spectral anisotropy.

Figure 3 illustrates the way in which the immobilized line shape of the CATSL can be obtained by spectral subtractions. Subtracting $35 \%$ of the integrated intensity of the spectrum of the ADP/ATP carrier/egg PC-PE-CL recombinant at 16 ${ }^{\circ} \mathrm{C}$ (Figure 3a) by use of the spectrum in egg phosphatidylcholine liposomes alone (Figure 3d) yields an immobilized end point (Figure 3b). Since the intensity of the immobilized component varied for the different recombinants (ranging between $50 \%$ and $90 \%$ ), the above assignment could be confirmed independently by pairwise intersubtractions between the recombinants. A typical liposome-bound end point (Figure 3c) from such intersubtractions is compared with the liposome-bound CATSL spectrum (Figure 3d); the complemen-

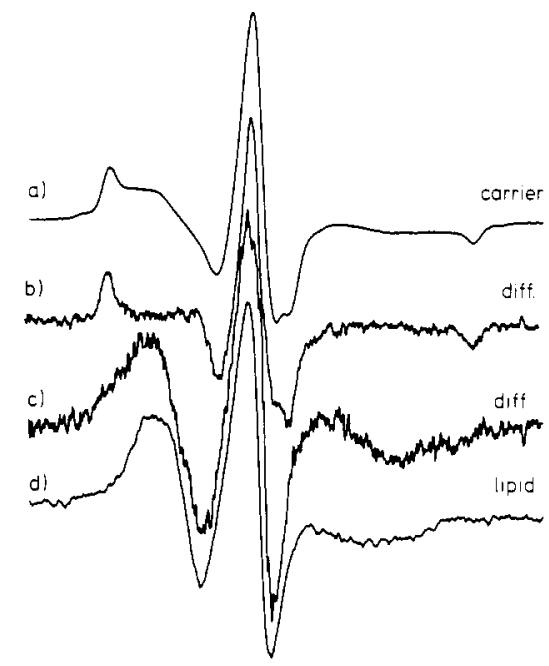

FIGURE 3: ESR difference spectroscopy of the spin-labeled carboxyatractylate label bound to reincorporated ADP/ATP carrier in phosphatidylcholine-phosphatidylethanolamine-cardiolipin liposomes, at $16^{\circ} \mathrm{C}$ : (a) ADP/ATP carrier/PC-PE-CL recombinant; (b) immobilized component difference spectrum obtained by subtracting the lipid spectrum (64\% of the double-integrated intensity) from the recombinant spectrum; (c) fluid component difference spectrum obtained by spectral intersubtractions between ADP/ATP carrier/PC and ADP/ATP carrier/PC-PE-CL spectra; (d) CATSL in egg phosphatidylcholine liposomes at $16^{\circ} \mathrm{C}$. Total scan range $=100 \mathrm{G}$.

tary immobilized end point was practically identical with that shown in Figure $3 b$.

The conventional ESR spectra of the immobilized CATSL display an unusually large spectral anisotropy and very narrow low-field and high-field extrema. This is characteristic of labels at the limits of motional sensitivity of conventional ESR spectroscopy, suggesting that there is little or no motion of the label relative to the protein and that the rotational motion of the protein as a whole may therefore be studied by saturation-transfer ESR spectroscopy. Nevertheless, the conventional spectra do display a slight sensitivity to environment. The values of the outer hyperfine splitting, $2 A_{\max }$, for CATSL in the solubilized and reincorporated ADP/ATP carrier are 70.3 and $71.1 \mathrm{G}$, whereas the corresponding values for spin-labeled maleimide in the same systems are 65.2 and $66.8 \mathrm{G}$, respectively. Essentially similar differences were observed in the line-width parameters, which unlike the outer splitting are not sensitive to hydrogen-bonding effects; for example, the low-field line widths in the reincorporated protein were 1.6 and $2.3 \mathrm{G}$ for CATSL and maleimide, respectively. Evidence for slow independent, segmental motion of the spin-label group relative to the protein has also been found in the immobilized spectrum of maleimide-labeled Na,K-ATPase (Esmann et al., 1987).

Representative second harmonic, $90^{\circ}$ out-of-phase, absorption STESR spectra of the maleimide spin label, the CATSL label, and the ATRSL label bound to Triton-solubilized, liposome-reincorporated, and native mitochondrial ADP/ATP carrier are shown in Figure 4. Saturation-transfer ESR of the CATSL spin label in the different environments and reconstitutions, corresponding to the conventional spectra in Figure 1, are given in Figure 5. These spectra are complicated by the presence of mobile labeled groups for the 5-MSL label (the sharp second-derivative lines in Figure 4a) and by the presence of the mobile lipid-bound component for the CATSL and ATRSL labels (cf. Figures 2 and 3 ) in Figures 4 and 5 . These mobile components overlap the diagnostic regions for the line-height analysis normally used in determining rotational correlation times from STESR spectra (Thomas et al., 1976) and necessitate use of the STESR in- 
Table I: STESR Normalized Total Integrals, $I_{\mathrm{b}}$, and Effective Rotational Correlation Times, $\tau_{\mathrm{R}}$, of the CATSL Spin Label in Triton-Solubilized, Liposome-Reincorporated, and Native Mitochondrial ADP/ATP Carrier ${ }^{a}$

\begin{tabular}{|c|c|c|c|c|c|c|}
\hline \multirow[b]{2}{*}{ sample } & \multicolumn{2}{|c|}{$0^{\circ} \mathrm{C}$} & \multicolumn{2}{|c|}{$15^{\circ} \mathrm{C}$} & \multicolumn{2}{|c|}{$30^{\circ} \mathrm{C}$} \\
\hline & $I_{b} \times 10^{2}$ & $\overline{\tau_{\mathrm{R}}(\mu \mathrm{s})}$ & $I_{\mathrm{b}} \times 10^{2}$ & $\tau_{R}(\mu \mathrm{s})$ & $I_{\mathrm{b}} \times 10^{2}$ & $\tau_{R}(\mu s)$ \\
\hline protein/Triton micelle & 0.28 & 5.5 & & & 0.15 & 3.0 \\
\hline protein/PC liposome & 0.61 & 59 & 0.48 & 27 & 0.48 & 27 \\
\hline protein/CL liposome & 0.56 & 44 & 0.43 & 20 & 0.30 & 6.8 \\
\hline protein/PC-PE liposome & 0.59 & 52 & 0.44 & 21 & 0.47 & 26 \\
\hline protein/PC-PE-CL liposome & 0.71 & 104 & 0.43 & 20 & 0.44 & 21 \\
\hline mitochondria & 0.89 & 287 & - & & 0.55 & 42 \\
\hline
\end{tabular}

${ }^{a}$ Each entry for the different samples corresponds to the mean for at least two independent experiments, obtained after accumulation of at least 12 spectra. Integrals were corrected for the presence of the mobile component with eq 1. Effective rotational correlation times (corresponding to the mean values of the corrected integrals) were determined by polynomial interpolations using calibrations for spin-labeled hemoglobin (Horvāth \& Marsh, 1988).

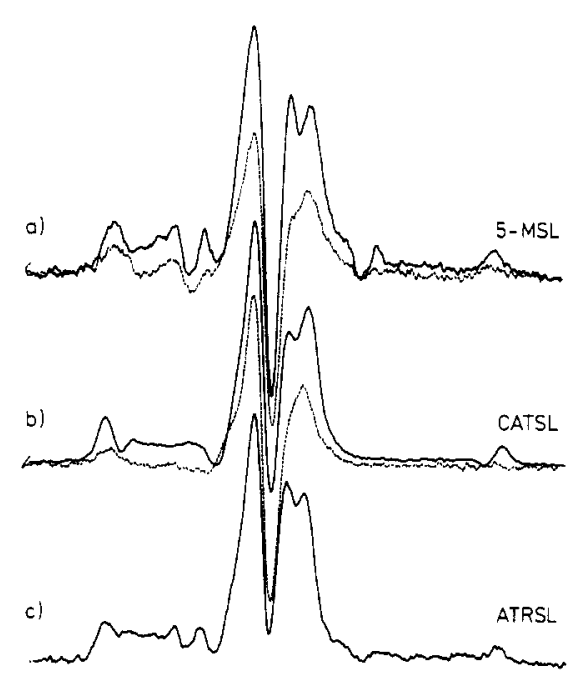

FIGURE 4: Second harmonic, $90^{\circ}$ out-of-phase, absorption STESR spectra ( $v_{2}^{\prime}$ display) of (a) maleimide- (5-MSL) labeled ADP/ATP carrier in PC-PE-CL (5.5:3.5:1 w/w) liposomes (full line) and in Triton micelles (dotted line), (b) CATSL spin label bound to carrier-containing PC-PE-CL liposomes (full line) and Triton micelles (dotted line), and (c) ATRSL spin label bound to mitochondria. Total scan range $=100 \mathrm{G}$

tegral method (Evans, 1981; Horvâth \& Marsh, 1983).

Second harmonic, $90^{\circ}$ out-of-phase, absorption STESR spectra of the CATSL in Triton-solubilized and liposomereincorporated carrier and in native mitochondria are given along with their integrals in Figure 6. These spectra were recorded at $0^{\circ} \mathrm{C}$. The first integrals of the STESR spectra are seen to increase progressively, corresponding to the changes in STESR line shape. For two-component spectra, the STESR integral intensities are linearly additive, and in the present case, the contribution of the second component is small because of its high mobility. The total STESR integral intensity $I_{\text {tot }}$ is therefore given by (Horvăth \& Marsh, 1983)

$$
I_{\text {tot }}=I_{\mathrm{b}} f_{\mathrm{b}}+I_{\text {lip }}\left(1-f_{\mathrm{b}}\right)
$$

where $I_{\mathrm{b}}$ and $I_{\text {lip }}$ are the intrinsic STESR intensities of the carrier-associated and lipid-associated spectra, respectively, and $f_{b}$ is the fraction of carrier-associated spin label which may be determined from spectral subtraction as indicated in Figure 3. All STESR integral intensities in eq 1 are normalized with respect to the second integral of the conventional spectra, i.e., to the respective amounts of CATSL in these two populations (Horvâth \& Marsh, 1983). The lipid-associated integral intensity can be determined from STESR spectra of the CATSL label in pure egg phosphatidylcholine liposomes and Triton micelles. None of these STESR spectra showed measureable integral intensity $\left(I_{\text {ves }}<0.03\right)$, and therefore, a value of $I_{\text {lip }}$ $=0$ was used in the subsequent calculations. Rotational

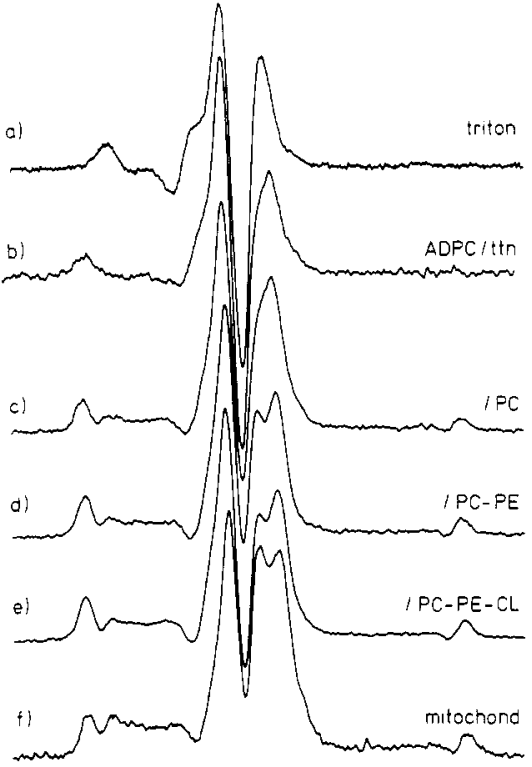

FIGURE 5: Second harmonic, $90^{\circ}$ out-of-phase, absorption STESR spectra ( $v_{2}^{\prime}$ display) of the CATSL spin label (a) in Triton micelles and bound to ADP/ATP carrier (b) in Triton micelles, (c) in PC liposomes, (d) in PC-PE (6:4 w/w) liposomes, (e) in PC-PE-CL $(5.5: 3.5: 1 \mathrm{w} / \mathrm{w})$ liposomes, and (f) in mitochondria. Total scan range $=100 \mathrm{G}$.
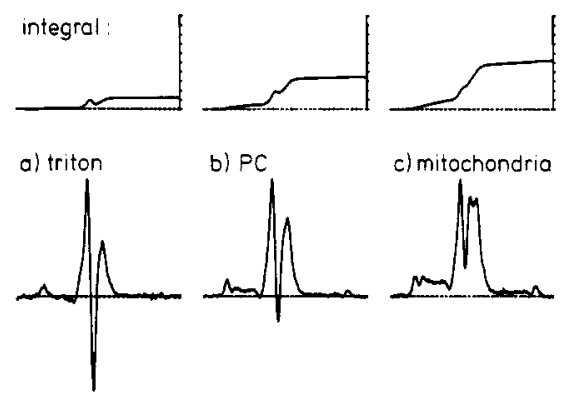

FIGURE 6: (Lower row) Second harmonic, $90^{\circ}$ out-of-phase, absorption STESR spectra ( $v_{2}^{\prime}$ display) of spin-labeled carboxyatractylate (a) bound to Triton-solubilized ADP/ATP carrier, (b) bound to reincorporated ADP/ATP carrier in phosphatidylcholine liposomes, and (c) bound to the native mitochondrial ADP/ATP carrier. (Upper row) First integrals of the STESR spectra in the lower row normalized with respect to the double-integrated intensity of the conventional spectra. (Full scale $=10^{-2}$, corresponding to an effective correlation time of $540 \mu \mathrm{s}$.) Total scan range $=100 \mathrm{G}$.

correlation times were obtained by polynomial interpolations from normalized STESR integral intensity vs correlation time calibration curves for spin-labeled hemoglobin (Horvăth \& Marsh, 1983, 1988).

The normalized STESR integral intensities, all corrected for the fraction $\left(1-f_{\mathrm{b}}\right)$ of mobile component, and the corresponding effective rotational correlation times are given for 
Table II: Rotational Correlation Times of the CATSL Spin Label in Triton-Solubilized, Liposome-Reincorporated, and Native Mitochondrial ADP/ATP Carrier, Deduced from the High-Field STESR Integrals and Line-Height Ratios ${ }^{a}$

\begin{tabular}{|c|c|c|c|c|c|c|}
\hline \multicolumn{7}{|c|}{ Part I: High-Field Normalized Integrals } \\
\hline \multirow[b]{2}{*}{ sample } & \multicolumn{2}{|c|}{$0^{\circ} \mathrm{C}$} & \multicolumn{2}{|c|}{$15^{\circ} \mathrm{C}$} & \multicolumn{2}{|c|}{$30^{\circ} \mathrm{C}$} \\
\hline & $I_{\mathrm{h}} \times 10^{3}$ & $\tau_{\mathrm{R}}(\mu \mathrm{s})$ & $I_{\mathrm{h}} \times 10^{3}$ & $\tau_{\mathrm{R}}(\mu \mathrm{s})$ & $I_{\mathrm{h}} \times 10^{3}$ & $\tau_{R}(\mu s)$ \\
\hline protein/Triton micelle & 0.11 & 2.9 & & & & \\
\hline protein/PC liposome & 0.18 & 10 & 0.13 & 4.6 & 0.08 & 0.9 \\
\hline protein/CL liposome & 0.19 & 11 & 0.15 & 6.7 & 0.07 & 0.5 \\
\hline protein/PC-PE liposome & 0.20 & 13 & 0.11 & 2.9 & 0.07 & 0.5 \\
\hline protein/PC-PE-CL liposome & 0.22 & 16 & 0.11 & 2.9 & 0.09 & 0.9 \\
\hline \multirow[t]{3}{*}{ mitochondria } & 0.40 & 39 & 0.18 & 10 & 0.11 & 2.9 \\
\hline & \multicolumn{4}{|c|}{ Part II: High-Field Line-Height Ratios } & \multirow{2}{*}{\multicolumn{2}{|c|}{$30^{\circ} \mathrm{C}$}} \\
\hline & \multicolumn{2}{|c|}{$0^{\circ} \mathrm{C}$} & \multicolumn{2}{|c|}{$15^{\circ} \mathrm{C}$} & & \\
\hline sample & $H^{\prime \prime} / H$ & $\overline{\tau_{R}(\mu s)}$ & $H^{\prime \prime} / H$ & $\overline{\tau_{\mathrm{R}}(\mu \mathrm{s})}$ & $H^{\prime \prime} / H$ & $\overline{\tau_{\mathrm{R}}(\mu \mathrm{s})}$ \\
\hline protein/Triton micelle & 0.14 & 2.1 & & & & \\
\hline protein/PC liposome & 0.22 & 5.9 & 0.14 & 2.1 & & \\
\hline protein/CL liposome & 0.21 & 5.5 & 0.13 & 1.7 & & \\
\hline protein/PC-PE liposome & 0.30 & 9.8 & 0.19 & 4.5 & 0.18 & 4 \\
\hline protein/PC-PE-CL liposome & 0.25 & 7.4 & 0.24 & 6.9 & 0.17 & 3.5 \\
\hline mitochondria & 0.39 & 14 & 0.32 & 11 & 0.17 & 3.5 \\
\hline
\end{tabular}

${ }^{a}$ Data sampling and integral evaluations as described for Table I.

the various forms of the ADP/ATP carrier in Table I. There are distinct trends in the data. The rotational correlation time at $0^{\circ} \mathrm{C}$ increases from 6 to $50-60 \mu$ s on going from the Triton micelle to the phospholipid liposomes and is still greater $(290$ $\mu \mathrm{s})$ for intact mitochondria. Very similar results were obtained for reconstitutions with the different lipid systems, except for the egg PC-PE-CL mixture at $0^{\circ} \mathrm{C}$. The effective rotational correlation times all decrease with increasing temperature, as expected, the temperature dependence being less pronounced for the protein/lipid recombinants because the lipids are in the fluid phase throughout the temperature range studied. The mitochondrial membrane (Hackenbrock et al., 1976), on the other hand, undergoes some kind of transition at ca. $14^{\circ} \mathrm{C}$ which is accompanied by considerable changes in the membrane viscosity. This also coincides with the point at which there is a change in slope in Arrhenius plots of the carrier transport activity (Klingenberg et al., 1982). At $30^{\circ} \mathrm{C}$ all three systems are in the fluid phase, and the differences between the rotational mobility of the ADP/ATP carrier in reincorporated systems and that in the intact mitochondrial membrane are smaller: all values lie in the range $\sim 20-40$ $\mu \mathrm{s}$. In Triton micelles, at the same temperature, the rotational rate is an order of magnitude faster.

The STESR integral calibrations on which these evaluations of the effective rotational correlation times are based are for isotropic motions. Anisotropic motion gives rise to differential responses in the three different diagnostic regions of the spectra (Marsh, 1980; Fajer \& Marsh, 1983). In analogy with the usual line-height ratio analysis, motional anisotropy can be detected by integration of selective regions in the spectra. A particularly useful region for such measurements is the high-field part of the spectrum because (i) this region is preferentially sensitive to $z$-axis wobbling or $(x, z)$ averaging, depending on the NO geometry with respect to the membrane fixed frame, and (ii) multicomponent spectra are best resolved in this region, allowing the selective measurement of immobilized components. From a comparison of the rotational correlation times derived from the total STESR integral and high-field integral calibrations (Horvăth \& Marsh, 1983) evidence can be obtained for motional anisotropy.

The results of high-field integration from the point $\mathrm{H}^{\prime \prime}, 1 / 3$ of the distance from the average $(x, y)$ position $\left[\theta=45^{\circ}, \phi\right.$ $=35^{\circ}$; see Horvatth and Marsh (1983) for definitions], to the end of the spectrum are summarized in Table II along with the high-field line-height ratios. As expected the rotational correlation times from the high-field integrals and the highfield line-height ratios agree reasonably closely. (It should be added that a few line-height ratios at $30^{\circ} \mathrm{C}$ were excluded from Table II because of poor signal-to-noise ratio; the selective integration from $\mathrm{H}^{\prime \prime}$ to the end of the spectrum gave reproducible results even in these cases because integration effectively averages the noise.) There are systematic differences, on the other hand, between the correlation times deduced from the total STESR integral and those deduced from the highfield region, the latter being lower in each case. This is consistent with saturation transfer due to $(x, z)$ averaging as a consequence of $y$-axis rotation. (Note that the $x$ and $y$ axes are interchangeable because of the near axial symmetry of the hyperfine anisotropy.) These differences between the effective correlation times determined from the total spectrum and from the high-field region parallel the difference found by Robinson and Dalton (1980) in simulations of highly anisotropic rotation where the nitroxide $z$ axis was oriented perpendicular to the rotation axis (see Discussion). The correlation time, $\tau_{20}$, for the total reorientation of the nitroxide $z$ axis was found to be a factor 10 greater than the effective correlation time, $\tau_{R}^{\text {eff }}$, determined from the high-field region. Thus our data strongly suggest that the CATSL label undergoes $y$-axis rotation while bound to the inhibitor binding site of the ADP/ATP carrier in liposome or membrane systems. In Triton, the difference between the effective correlation times deduced from the high-field and total integrals is much smaller, consistent with the rotation of the protein-containing micelles being more nearly isotropic (see Discussion).

The results of the experiments with the ATRSL label and with the maleimide-labeled protein are compared with those for CATSL in Table III. The atractylate spin label lacks one of the carboxylate moieties of the CATSL structure shown in structure I. From a comparison of the ATRSL and CATSL data in mitochondria, it is clear that the ATRSL is, if anything, more strongly held in the carrier binding site. This is in spite of lacking the carboxylic group and suggests that the binding is not entirely of electrostatic origin. It is interesting to note that CATSL, although not being more rigidly held in the inhibitor binding site, can displace bound ATR in CATSL/ ATR displacement experiments (Munding et al., 1983).

Covalent spin labeling by 5 -MSL requires rearrangement of the ATR-carrier complex from the $C$ conformation into the $\mathrm{M}$ conformation, by addition of bongkrekic acid and ADP (Munding et al., 1987). It has been shown previously that this 
Table III: Comparison of Rotational Correlation Times of the Carboxyatractylate, Atractylate, and 5-Maleimide Spin Labels in Triton-Solubilized, Liposome-Reincorporated, and Native Mitochondrial ADP/ATP Carrier ${ }^{a}$

\begin{tabular}{|c|c|c|c|c|c|c|}
\hline \multirow[b]{2}{*}{ sample } & \multicolumn{2}{|c|}{ CATSL } & \multicolumn{2}{|c|}{ ATR } & \multicolumn{2}{|c|}{ 5-MSL } \\
\hline & $I_{b} \times 10^{2}$ & $\overline{\tau_{R}(\mu s)}$ & $I_{\mathrm{b}} \times 10^{2}$ & $\overline{\tau_{\mathrm{R}}(\mu \mathrm{s})}$ & $\overline{I_{\mathrm{b}} \times 10^{2}}$ & $\tau_{\mathrm{R}}(\mu \mathrm{S})$ \\
\hline \multicolumn{7}{|c|}{ STESR Normalized Integrals at $0^{\circ} \mathrm{C}$} \\
\hline protein/Triton micelle & 0.28 & 5.5 & & & 0.14 & 0.2 \\
\hline protein/PC-PE-CL liposome & 0.71 & 104 & & & 0.31 & 7.6 \\
\hline mitochondria & 0.89 & 287 & 0.93 & 362 & & \\
\hline \multicolumn{7}{|c|}{ STESR Normalized Integrals at $30^{\circ} \mathrm{C}$} \\
\hline protein/Triton micelle & 0.15 & 3.0 & & & 0.11 & $<0.1$ \\
\hline protein/PC-PE-CL liposome & 0.44 & 21 & & & 0.21 & 1.8 \\
\hline mitochondria & 0.55 & 42 & 0.64 & 70 & & \\
\hline
\end{tabular}

${ }^{a}$ Data sampling and integral evaluation as described for Table I.

rearrangement leads to unmasking of the maleimide binding sites (Aquila \& Klingenberg, 1982; Leblanc \& Klauser, 1972; Vignais \& Vignais, 1972). Although the labeled protein is therefore in a different conformation from that binding the ATRSL and CATSL labels, it is unlikely that the large differences seen in Table III can be attributed solely to this source. The correlation times for covalently bound 5-MSL are almost an order of magnitude lower, which is an indication of segmental motion in the maleimide binding region of the protein.

\section{Discussion}

The experiments described above were conducted with the primary aim of studying the rotational diffusion of the ADP/ATP carrier protein, both in the natural and in reincorporated systems. It is clear that the maleimide spin label is unsuitable for such studies because its STESR spectrum largely reflects segmental mobility of the protein. The CATSL and ATRSL labels exhibit an unusually strong degree of immobilization when bound to the carrier (Munding et al., 1983), the conventional ESR spectra lying close to the rigid limit (cf. Figure 1). Thus these noncovalent labels are particularly well-suited to studies of the rotational motion of the protein as a whole. The high affinity of the inhibitors $\left(K_{\mathrm{D}} \sim 10 \mathrm{nM}\right)$ means that there is no interference from an aqueous spin-label component, and the high specificity means that the ADP/ATP carrier can be studied selectively in the presence of the other proteins of the inner mitochondrial membrane. Complications from spectral components due to labels in the lipid phase of the membrane can be handled by the STESR integral method (Evans, 1981; Horvâth \& Marsh, 1983). Further analysis of the membrane- or liposome-bound form of the carrier, however, requires consideration of the anisotropic nature of the protein rotational diffusion.

Hydrodynamic measurements on the carrier in Triton (Hackenberg \& Klingenberg, 1980) suggest that the STESR measurements will reflect the rotational diffusion of the protein-containing micelle as a whole. Tables I and II show that the high-field and total STESR integrals yield rather similar values for the correlation time, consistent with a near isotropic rotation, which is to be expected for the whole micelle. The effective correlation time for isotropic rotation can be obtained from the Debye equation: $\tau_{\mathrm{R}}=4 \pi \eta r^{3} /(3 k T)$, where $r$ is the effective micellar radius and $\eta$ is the viscosity of the medium. Taking the Stokes radius of $65 \AA$ from translational diffusion measurements (Hakenberg \& Klingenberg, 1980) yields an effective correlation time of $0.3 \mu \mathrm{s}$ for $\eta=1 \mathrm{cP}$. The frictional ratio obtained from the translational diffusion measurements is $f / f_{0}=1.56$, which implies frictional ratios of $\approx 5$ for the rotational diffusion of an oblate ellipsoid of equivalent axial ratio (Koenig, 1975). Hence, the predicted correlation time for rotational diffusion is $\tau \sim 1.5 \mu$ s. Comparison with the results of Table I shows that the measured values are a factor of 2-4 greater than this estimate. However, the viscosity of the concentrated Triton solution is certainly considerably greater than the value of $\eta=1 \mathrm{cP}$ for pure water, which most probably accounts for the difference in the two values. A similar effect has been observed in the highly concentrated detergent solutions used in STESR measurements on the rotational diffusion of the Na,K-ATPase (Esmann et al., 1987). Thus the rotational diffusion measurements on the micellar solution of the carrier are reasonably consistent with those obtained previously for translational diffusion, in which it was demonstrated by both sedimentation-diffusion and sedimentation-equilibrium studies that the carrier was in a dimeric form (Hakenberg \& Klingenberg, 1980). A protein dimer was also found for the carrier in a different detergent, by neutron scattering techniques (Block et al., 1982). Measurements of the effective molecular weight per binding site (Riccio et al., 1975 ) and cross-linking experiments (Hackenberg \& Klingenberg, 1980) have further shown that the AAC is in a dimeric state.

Comparison of the data from the high-field integral for the membrane- and liposome-bound carrier (see Table II) with that from the total integral (see Table I) indicates that the carrier is undergoing highly anisotropic rotational diffusion and that the $z$ axis of the spin-label nitroxide group must have an orientation $\left(\theta \approx 90^{\circ}\right)$ which is close to perpendicular to the rotation axis of the protein (assumed to be the membrane normal). In this connection it should be noted that there is one unique spin-labeled inhibitor binding site per protein dimer. Robinson and Dalton (1980) have made simulations of the first harmonic dispersion STESR spectra for anisotropic rotation with the $\theta=90^{\circ}$ spin-label orientation. They related the effective rotational correlation time, $\tau_{\mathrm{R}}^{\text {eff }}$, deduced from isotropic model systems, to the true value of the correlation time for the uniaxial rotation. The latter is defined by $\tau_{\mathrm{R} \|}=1$ / $6 D_{\mathrm{R} \|}$, where $D_{\mathrm{R} \|}$ is the rotational diffusion coefficient. For highly anisotropic rotation $\left(D_{\mathrm{R} \|} \gg D_{\mathrm{R}_{\perp}}\right)$ where the reorientation of the major axis was extremely slow $\left(D_{\mathrm{R} \perp} \approx 0\right)$, it was found that the effective isotropic correlation time was longer than the true correlation time according to the relation $\tau_{\mathbf{R}}^{\text {eff }}=$ $2 \tau_{\mathrm{R} \|}$. Reference to the data of Table II therefore suggests that values of $\tau_{\mathrm{R} \|}$ for the carrier lie in the region of ca. 1-5 $\mu \mathrm{s}$ in phospholipid liposomes and ca. 2-20 $\mu \mathrm{s}$ in mitochondria, at temperatures of 30 and $0^{\circ} \mathrm{C}$, respectively. It will be noted that these are the maximum values which $\tau_{\mathrm{R} \mid}$ may take. If the orientation, $\theta$, of the nitroxide $z$ axis is less than $90^{\circ}$ to the diffusion axis, the STESR spectra will be less sensitive to the axial diffusion [cf. Marsh (1980) and Robinson and Dalton (1980)], and $\tau_{R \|}$ will be overestimated.

Information regarding the state of aggregation of the protein in the membrane can, in principle, be determined from comparison of the experimental values of $\tau_{R \|}$ with theoretical 
predictions of the rotational diffusion coefficient, $D_{\mathbf{R} \| \text {. An }}$ expression for the rotational diffusion coefficient of a cylindrical protein, predicted from hydrodynamic theory, has been given by Saffman and Delbrueck (1975), and the extension to proteins of elliptical cross section has been given by Jähnig (1986):

$$
D_{\mathrm{R} \|}=\frac{k T}{4 \pi \eta a b h} \frac{2 a / b}{1+(a / b)^{2}}
$$

where $a$ and $b$ are the semiaxes of the cylinder $(a>b), h$ is the height of the membrane-spanning region of the cylinder, $\eta$ is the effective viscosity within the membrane, $k$ is Boltzman's constant, and $T$ is the absolute temperature.

Values of $a=23 \AA$ and $b=20 \AA$ for the protein dimer can be estimated from the hydrodynamic model obtained by Hackenberg and Klingenberg (1980), if it is assumed that the cross section of the dimer has the same asymmetry $(a / b=$ $65 / 57$ ) as that induced in the protein-containing Triton micelle. ${ }^{2}$ For $h=45 \AA$ and values of the effective membrane viscosity within the realistic range $\eta=2.4-5 \mathrm{P}$ [see, e.g., Cherry and Godfrey (1981)], the calculated rotational correlation time for the dimer varies from $\tau_{R \|}=2.5$ to $\tau_{R \|}=6$ $\mu \mathrm{s}$. Alternatively, if it is assumed that the dimer volume is accommodated solely within the membrane with a height of $45 \AA$, then $a=25 \AA$ and $b=22 \AA$ and the corresponding range of values for $\tau_{\mathrm{R} \|}$ is increased to $3-7 \mu \mathrm{s}$. It is seen that these values agree reasonably well with the measurements of the rotational correlation time in lipid liposomes, deduced from the high-field region of the spectrum, but the measurements in mitochondria at $0^{\circ} \mathrm{C}$ lie outside the range predicted for a dimer. Thus it seems probable that the protein is present predominantly as a dimer in the reincorporated system, but either is more highly aggregated or, more likely is interacting with other proteins in the mitochondrial membrane. The finding of a minimally dimeric state of aggregation of the carrier is in good agreement with studies on inhibitor binding, which is found to have a stoichiometry of a single carboxyatractyloside molecule per dimer (Riccio et al., 1975) and has been suggested to be an essential feature of the carrier mechanism (Klingenberg, 1981). An oligomeric state (most probably dimers) has previously been proposed for the native carrier in proteoliposomes, on the basis of electron microscopy data (Brandolin et al., 1980).

Previous measurements on the rotational diffusion of the ADP/ATP carrier in mitochondria, submitochondrial particles, and proteoliposomes have been reported with transient dichroism (Müller et al., 1982, 1984). Rotational relaxation times of $405 \mu \mathrm{s}$ at $20^{\circ} \mathrm{C}$ in mitochondria, $240 \mu \mathrm{s}$ at $5^{\circ} \mathrm{C}$ in submitochondrial particles, and $246 \mu \mathrm{s}$ in proteoliposomes were found, corresponding to rotational correlation times of 67,40 , and $41 \mu \mathrm{s}$, respectively. These results, however, must be taken with considerable reservation. First, it has been shown that labeling of the protein in mitochondria with eosin maleimide results to a large extent in the labeling of other proteins (Finckh, 1987). This background labeling is evident only on appropriate exposure of the gels. The reported decay of transient dichroism therefore reflects the average mobility of labeled mitochondrial proteins, of which the fluorescence due to the ADP/ATP carrier represents at the most 50\%. Similar considerations can also be expected to apply to the results with submitochondrial particles. Finally, the activity of the pro-

\footnotetext{
${ }^{2}$ These values were calculated from the total volume of the dimer molecule of molecular weight 64000 and partial specific volume 0.73 , together with a total height of the protein of $54 \AA$ and cross-sectional asymmetry of $a / b=65 / 57$ (Hackenberg \& Klingenberg, 1980).
}

teoliposomes used in the above-quoted studies was not clearly characterized. This is crucial because the bongrekate-protein-eosin maleimide complex is extremely unstable and results in protein denaturation (Aquila et al., 1978; Kramer et al., 1977). For these reasons, the dichroism data cannot be directly compared with the present results on the stable CAT-protein complex. Further, the ambiguity in the eosin maleimide labeling is circumvented by spin labeling with the specific probe CATSL.

In summary, the STESR measurements suggest that the ADP/ATP carrier undergoes anisotropic rotational diffusion, which is much slower in mitochondria than in reconstituted phospholipid liposomes. No large differences were found in the protein mobility in different lipid environments, with the possible exception of the cardiolipin-containing system at low temperature. Interestingly, a preference for certain negatively charged lipids (including cardiolipin) has been found in experiments on lipid/protein interactions in these systems (Horvăth, Drees, Beyer, Klingenberg, and Marsh, unpublished results; Beyer \& Klingenberg, 1985; Drees and Beyer, unpublished results).

\section{REFERENCES}

Aquila, H., \& Klingenberg, M. (1982) Eur. J. Biochem. 122, 141-145.

Aquila, H., Eiermann, W., Babel, W., \& Klingenberg, M. (1978) Eur. J. Biochem. 85, 549-560.

Beyer, K. (1982) J. Colloid Interface Sci. 86, 73-89.

Beyer, K., \& Klingenberg, M. (1983) Biochemistry 22, 639-645.

Beyer, K., \& Klingenberg, M. (1985) Biochemistry 24, $3821-3826$.

Blair, P. V. (1967) Methods Enzymol. 10, 78-81.

Block, M. R., Zaccai, G., Lauquin, G. J. M., \& Vignais, P. V. (1982) Biochem. Biophys. Res. Commun. 109, 471-477.

Brandolin, G., Doussiere, J., Gulik, A., Gulik-Krzywicki, T., Lauquin, G. J. M., \& Vignais, P. V. (1980) Biochim. Biophys. Acta 592, 592-614.

Cherry, R. J., \& Godfrey, R. E. (1981) Biophys. J. 36, 257-276.

Esmann, M., Horvăth, L. I., \& Marsh, D. (1987) Biochemistry 26, 8675-8683.

Evans, C. A. (1981) J. Magn. Reson. 49, 109-116.

Fajer, P., \& Marsh, D. (1982) J. Magn. Reson, 49, 212-224.

Fajer, P., \& Marsh, D. (1983) J. Magn. Reson. 51, 446-459.

Finckh, P. (1987) Doctoral Thesis, Universität München.

Hackenberg, H., \& Klingenberg, M. (1980) Biochemistry 19, 546-555.

Hackenbrock, C. R., Höchli, M., \& Chau, R. M. (1976) Biochim. Biophys. Acta 455, 466-484.

Hemminga, M. A., de Jager, P. A., Marsh, D., \& Fajer, P. (1984) J. Magn. Reson. 59, 160-163.

Horvăth, L. I., \& Marsh, D. (1983) J. Magn. Reson. 54, 363-373.

Horvăth, L. I., \& Marsh, D. (1988) J. Magn. Reson. (in press).

Jähnig, F. (1986) Eur. J. Biophys. 14, 63-64.

Klingenberg, M. (1981) Nature (London) 290, 449-454.

Klingenberg, M. (1985) in The Enzymes of Biological Membranes (Martonosi, A. N., Ed.) Vol. 4, pp 511-553, Plenum, New York.

Klingenberg, M., Grebe, K., \& Appel, H. (1982) Eur. J. Biochem. 126, 263-269.

Koenig, S. (1975) Biopolymers 14, 2421.

Kramer, R., Aquila, H., \& Klingenberg, M. (1977) Biochemistry 16, 4949-4953. 
Lauquin, G. J. M., Devaux, P. F., Bienvenue, A., Villiers, C., \& Vignais, P. V. (1977) Biochemistry 16, 1202-1208.

Leblanc, P., \& Clauser, H. (1972) FEBS Lett. 23, 107-1 13.

Marsh, D. (1980) Biochemistry 19, 1632-1637.

Müller, M., Krebs, J. J. R., Cherry, R. J., \& Kawato, S. (1982) J. Biol. Chem. 257, 1117-1120.

Müller, M., Krebs, J. J. R., Cherry, R. J., \& Kawato, S. (1984) J. Biol. Chem. 259, 3037-3043.

Munding, A., Beyer, K., \& Klingenberg, M. (1983) Btochemistry 22, 1941-1947.

Munding, A., Drees, M., Beyer, K., \& Klingenberg, M. (1987) Biochemistry 26, 8637-8644.
Riccio, P., Aquila, H., \& Klingenberg, M. (1975) FEBS Lett. 56, 133-138.

Robinson, B. H., \& Dalton, L. R. (1980) J. Chem. Phys. 72, 1312-1324.

Saffman, P. G., \& Delbrueck, M. (1975) Proc. Natl. Acad. Sci. U.S.A. 72, 3111-3113.

Thomas, D. D., Dalton, L. R., \& Hyde, J. S. (1976) J. Chem. Phys. 65, 3006-3024.

Vignais, P. V., \& Vignais, P. M. (1972) FEBS Lett. 26, $27-31$.

Wells, M. A., \& Hanahan, D. J. (1969) Methods Enzymol. 14, 179-183. 